\title{
Association between the high-dose use of benzodiazepines and rehospitalization in patients with schizophrenia: a 2-year naturalistic study
}

This article was published in the following Dove Press journal:

Neuropsychiatric Disease and Treatment

15 December 2016

Number of times this article has been viewed

\author{
Yukika Takita' \\ Yoshikazu Takaesu' \\ Kotaro Ono' \\ Kunihiro Futenma' \\ Akiyoshi Shimura' \\ Akiko Murakoshi' \\ Yoko Komada ${ }^{2}$ \\ Yuichi Inoue ${ }^{1,2}$ \\ Takeshi Inoue' \\ 'Department of Psychiatry, \\ ${ }^{2}$ Department of Somnology, Tokyo \\ Medical University, Tokyo, Japan
}

Correspondence: Yoshikazu Takaesu

Department of Psychiatry, Tokyo

Medical University, 6-7-I Nishishinjuku,

Shinjuku-ku, Tokyo 160-0023, Japan

$\mathrm{Tel}+8 \mid 333426$ III

Fax +8I 333427083

Email esu-yosh@tokyo-med.ac.jp
Background: High-dose use of benzodiazepines (BZPs) reportedly causes adverse effects on cognitive function and quality of life in patients with schizophrenia. However, effects of BZPs on the clinical course of schizophrenia have not been clarified. This study was set out to investigate the association between BZPs and rehospitalization of patients with schizophrenia.

Methods: In this retrospective study, patients with schizophrenia who were discharged from Tokyo Medical University Hospital between January 2009 and February 2012 were eligible as subjects. One hundred and eight patients who continued treatment for $>2$ years after hospital discharge were included in this study. Clinical characteristics, doses of prescribed medication such as BZPs and antipsychotics, and Global Assessment of Functioning scores at discharge were investigated. The primary outcome was rehospitalization of patients for any reason.

Results: In a total of 108 subjects with schizophrenia, 44 subjects (40.7\%) experienced rehospitalization during the 2-year study period. A multivariate analysis by the Cox proportional hazards model revealed that low educational history (hazard ratio $=2.43, P=0.032$ ), younger onset age of schizophrenia (hazard ratio $=2.10, P=0.021$ ), and higher diazepam-equivalent dose (hazard ratio $=6.53$, $P=0.011$ ) were significantly associated with the time to rehospitalization after hospital discharge.

Conclusion: The results of this study suggest that high-dose use of BZPs at discharge in patients with schizophrenia might be associated with a shorter time to rehospitalization.

Keywords: schizophrenia, rehospitalization, risk factor, high-dose benzodiazepine, Cox proportional hazards model

\section{Introduction}

Schizophrenia is a chronic psychiatric disease that has frequent relapses during the clinical course. ${ }^{1}$ A Cochrane database systematic review reported that $27 \%$ of schizophrenia patients experienced relapse within 12 months, even though they continued antipsychotic medication. ${ }^{2}$ Previous studies have also pointed out several risk factors for relapse, such as nonadherence to antipsychotics, ${ }^{3}$ interruption of treatment, ${ }^{1,4}$ lower quality of life (QOL), ${ }^{5}$ decreased cognitive function, ${ }^{6}$ poor premorbid adaptation to school, ${ }^{2}$ premorbid social withdrawal, ${ }^{2}$ shorter duration after the onset of schizophrenia, ${ }^{7}$ and involuntary hospitalization. ${ }^{8}$ A study with a long-term follow-up for $\geq 20$ years revealed that the younger onset age of schizophrenia was significantly associated with the risk of relapse, while sex and marital status were not associated with relapse. ${ }^{9}$ Moreover, substance abuse, including benzodiazepines (BZPs) that were used as adjunctive medication for patients with schizophrenia, has been reported to increase the risk of relapse. ${ }^{7}$

BZPs are widely used in clinical settings as adjunctive treatment for psychiatric diseases including schizophrenia. However, adverse effects of BZPs, especially with 
high-dose use, have been pointed out by several studies, such as risk of falling, fractures, ${ }^{10}$ nocturnal abnormal behavior, ${ }^{11}$ and risk of dependence. ${ }^{12,13}$ Since there has been no evidence about efficacy of BZPs in patients with schizophrenia as adjunctive treatment together with antipsychotics, this kind of drug is recommended only for short-term use for insomnia and anxiety. ${ }^{14}$ Nevertheless, $71 \%-86 \%$ of the patients with schizophrenia receive adjunctive treatment with BZPs in Japan; this rate seems to be higher than that in the United States. ${ }^{15}$ A recent research article reported that cognitive function and QOL were improved after reducing the amount of BZPs in patients with schizophrenia. ${ }^{15}$ Taking these studies into consideration, long-term and high-dose use of BZPs might affect the clinical course of schizophrenia. However, apparently no study focused on this issue.

Therefore, a naturalistic observational study focused on the relationship between high-dose BZPs and the clinical course of patients with schizophrenia for 2 years after hospital discharge was conducted. In addition, rehospitalization was targeted as a primary outcome in this study, which was a clear and clinically important indication of relapse in patients with schizophrenia.

\section{Materials and methods Subjects}

The subjects were selected from the Tokyo Medical University Hospital from January 2009 to February 2012. The subjects were diagnosed as having schizophrenia based on the diagnostic criteria of the Diagnostic and Statistical Manual of Mental Disorders (DSM-IV-TR) by at least two specialists in psychiatry. Patients who continued to have treatment in the outpatient clinic for $\geq 2$ years were included. The cutoff period of this study was chosen as 2 years according to a previous study. ${ }^{16}$ Patients were excluded if they met the following criteria: 1) patients diagnosed with schizoaffective disorder or persistent delusional disorder; 2) patients who had physical diseases that might have an impact on the course of schizophrenia, including collagen disease, brain injury/stroke, dementia, and diabetes; and 3) those with a history of drug or alcohol abuse within the past 12 weeks. This study was conducted with the approval of the ethics committee of Tokyo Medical University which waived the requirement to obtain patient's consent due to the retrospective nature of the study.

\section{Procedures}

Demographic and clinical parameters of the subjects, including age at the time of investigation, age at the onset of schizophrenia, sex, family members, marital status, educational history, working years, duration of illness of schizophrenia, length of stay at the last hospitalization, Global Assessment of Functioning (GAF) at the time of discharge, types of antipsychotics at the time of discharge, total doses of antipsychotics at the chlorpromazine (CP)equivalent dose at the time of discharge, and total doses of BZP at the diazepam (DZP)-equivalent dose at the time of discharge were evaluated.

\section{Statistical analysis}

At first, the subjects were divided into two groups: the rehospitalized group, who were rehospitalized within 2 years after discharge from the previous hospitalization, and the non-rehospitalized group. Student's $t$-test and the chi-squared test were used to compare the demographic and clinical parameters between the two groups. The Kaplan-Meier method was used to calculate the cumulative survival rates of the subjects. Analysis with the Cox proportional hazards model was performed for the factors associated with time to rehospitalization. All variables were analyzed as categorical variables. Continuous variables, including age at the time of investigation, age at the onset of schizophrenia, working years, duration of schizophrenia morbidity, duration of hospitalization, GAF, CP-equivalent dose of antipsychotics, and DZP-equivalent dose of BZPs, were divided by the median values and analyzed as categorical variables. In the Cox proportional hazard analysis, variables that showed significant association in univariate analyses were put into a multivariate model. SPSS Version 19 (SPSS Inc., Chicago, IL, USA) was used for all the statistical analyses. The significance level was set at $P<0.05$.

\section{Results}

One hundred and ninety-three patients aged 18-65 years who were discharged from the hospital met the inclusion criteria for schizophrenia. Finally, 108 patients $(55 \%)$ who continued to have treatment in the outpatient clinic for $\geq 2$ years were included in this study. In 108 subjects with schizophrenia, 44 subjects $(40.7 \%)$ experienced rehospitalization during the 2-year study period. The Kaplan-Meier method revealed cumulative survival rates (Figure 1).

Comparison of demographic and clinical parameters between the rehospitalized group and non-rehospitalized group is shown in Table 1. Age at the time of discharge and age at the onset of schizophrenia in the rehospitalized group were significantly lower than those in the non-rehospitalized group. The rate of the number of patients with college graduation in the rehospitalized group was significantly lower than 


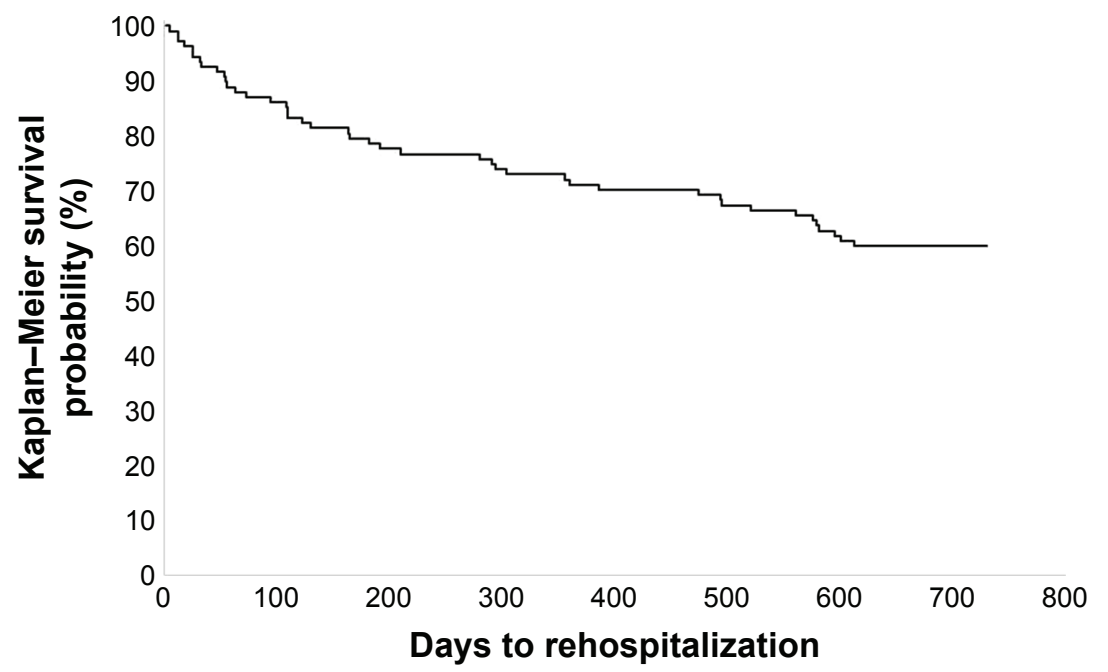

Figure I Kaplan-Meier analysis of days to rehospitalization within 2 years.

that in the non-rehospitalized group. Lifetime working years in the rehospitalized group was significantly shorter than that in the non-rehospitalized group. The $\mathrm{CP}$-equivalent dose and DZP-equivalent dose in the rehospitalized group were significantly higher than those in the non-rehospitalized group.

Univariate analyses of the Cox proportional hazard model revealed that age at the time of discharge, age at the onset of schizophrenia, college graduation, lifetime working years, CP-equivalent dose, and DZP-equivalent dose were significantly associated with rehospitalization after discharge. Multivariate analysis by the Cox proportional hazards model with step-wise method revealed that low educational history, younger age of onset of schizophrenia, and higher DZPequivalent dose were significantly associated with time to rehospitalization after discharge (Table 2 and Figure 1).

\section{Discussion}

This may be the first study to reveal the relationship between the high-dose usage of BZPs and the clinical course of patients with schizophrenia. The result of this study showed that high-dose use of BZPs ( $>10 \mathrm{mg}$ DZP-equivalent dose), lower educational history, and younger onset age of schizophrenia were significantly associated with time to rehospitalization. On the other hand, lower-dose use of BZPs ( $<10 \mathrm{mg})$ was not associated with the duration of rehospitalization. Our previous studies suggested that BZPs lead to abnormal behavior during sleep ${ }^{11}$ and risk of dependency on BZPs ${ }^{12}$ in schizophrenic patients. In addition to these adverse effects of BZPs on schizophrenia, the results of this study suggest that BZPs might worsen the clinical course of schizophrenia (relapse in particular) as the dose increases.

Table I Comparison of demographic and clinical parameters of the subjects between the rehospitalized group and non-rehospitalized group

\begin{tabular}{|c|c|c|c|c|}
\hline Variables & $\begin{array}{l}\text { Overall } \\
(n=108)\end{array}$ & $\begin{array}{l}\text { Rehospitalized } \\
(n=44)\end{array}$ & $\begin{array}{l}\text { Non-rehospitalized } \\
(n=64)\end{array}$ & P-value \\
\hline Age at the time of discharge, years & $44.1 \pm 16.3$ & $38.1 \pm 13.9$ & $48.1 \pm 16.7$ & 0.001 \\
\hline Onset age of schizophrenia, years & $29.9 \pm 14.0$ & $25.2 \pm 11.0$ & $33.0 \pm 14.9$ & 0.003 \\
\hline Sex (male:female) & $30: 78$ & $9: 35$ & $21: 43$ & 0.193 \\
\hline Living with family (yes:no) & $80: 28$ & $37: 7$ & $4: 21$ & 0.073 \\
\hline Marital status (married:unmarried) & $25: 83$ & $9: 35$ & $16: 48$ & 0.648 \\
\hline College graduation (yes:no) & $32: 76$ & $7: 37$ & $25: 39$ & 0.011 \\
\hline Life-time working years & $8.8 \pm 11.9$ & $5.5 \pm 7.8$ & $11.1 \pm 13.7$ & 0.010 \\
\hline Duration of schizophrenia, years & $15.0 \pm 13.2$ & $14.1 \pm 12.5$ & $15.6 \pm 13.8$ & 0.539 \\
\hline Length of stay, days & $44.7 \pm 51.4$ & $48.3 \pm 68.0$ & $42.3 \pm 36.3$ & 0.593 \\
\hline GAF scores at the time of discharge, points & $56.6 \pm 12.8$ & $55.4 \pm 9.8$ & $57.3 \pm 14.4$ & 0.400 \\
\hline $\mathrm{CP}$-equivalent dose at the time of discharge, $\mathrm{mg}$ & $506.1 \pm 364.3$ & $609.7 \pm 416.3$ & $434.9 \pm 307.5$ & 0.020 \\
\hline DZP-equivalent dose at the time of discharge, $\mathrm{mg}$ & $12.3 \pm 11.2$ & $18.5 \pm 12.4$ & $8.0 \pm 7.8$ & $<0.001$ \\
\hline
\end{tabular}

Notes: The values represent the mean \pm SD. Student's $t$-test was used to compare the continuous variables between the two groups. The chi-squared test was used to compare the categorical variables.

Abbreviations: GAF, Global Assessment of Functioning; CP, chlorpromazine; DZP, diazepam. 
Table 2 Multivariate Cox hazard proportional analysis for factors associated with time to rehospitalization

\begin{tabular}{|c|c|c|}
\hline Variables & Hazard ratio $(95 \% \mathrm{Cl})$ & $P$-value \\
\hline \multicolumn{3}{|c|}{ College graduation } \\
\hline Yes & (ref) & \\
\hline No & $2.43(1.08-5.48)$ & 0.032 \\
\hline \multicolumn{3}{|c|}{ Age at the onset, years } \\
\hline$\geqq 25$ & (ref) & \\
\hline$<25$ & $2.10(1.12-3.94)$ & 0.021 \\
\hline \multicolumn{3}{|c|}{ DZP-equivalent dose, $\mathrm{mg}$} \\
\hline 0 & (ref) & \\
\hline$<10$ & $2.73(0.62-11.10)$ & NS \\
\hline$\geqq 10$ & $6.54(1.54-27.73)$ & 0.011 \\
\hline
\end{tabular}

Abbreviations: NS, not significant; DZP, diazepam; ref, reference; $\mathrm{Cl}$, confidence interval.

The causal relationship between high-dose usage of BZPs and rehospitalization in patients with schizophrenia was unclear in this study. However, a previous study suggested a negative effect of high-dose use of BZPs on patients with schizophrenia. Kitajima et $\mathrm{al}^{15}$ investigated the effect of reducing BZP doses on cognitive function of schizophrenic patients for 8 weeks and revealed that cognitive function was improved significantly in them after reducing BZP doses, compared with the control patients. It is possible that highdose use of BZPs reduces cognitive function, consequently leading to worsening symptoms of schizophrenia. In addition, a large case-control study suggested that concomitant usage of BZP was related to the risk of death in schizophrenic patients. ${ }^{17}$ Similarly, it was reported that concomitant use of BZP with intramuscular depot formulations of olanzapine possibly increased the death rate in schizophrenia patients. ${ }^{18}$ Therefore, psychiatrists should pay attention to concomitant use of BZP for schizophrenia patients. Finally, there is a possibility that BZP usage may lower the adherence to antipsychotic medication, although apparently no study has investigated this issue.

On the other hand, it is also possible that schizophrenic patients with severe symptoms, including insomnia and anxiety, which are target symptoms of BZPs, are likely to have high doses of BZPs. ${ }^{19}$ However, considering the result of our study that GAF scores at hospital discharge were not significantly associated with rehospitalization, high-dose use of BZPs might affect the clinical course of schizophrenia. Although the results of this study could not clarify how highdose usage of BZPs affects the clinical course of schizophrenia, clinicians should avoid using high-dose BZPs because previous studies have suggested that BZPs did not improve any clinical outcome of schizophrenia. ${ }^{14}$
The results of this study pointed out that younger onset age of schizophrenia and lower educational history were also factors associated with time to rehospitalization, which were similar to the results of previous studies. Eaton et $\mathrm{al}^{9}$ suggested that lower onset age of schizophrenia was associated with rehospitalization after the initial hospital discharge, as a result of multivariate Cox proportional analysis for $>20$ years follow-up. ${ }^{9}$ In addition, Suzuki et $\mathrm{al}^{20}$ performed a multiple logistic regression analysis for factors associated with rehospitalization in 67 outpatients with schizophrenia and revealed that lower educational history was significantly associated with rehospitalization. The results of these two studies were in line with the results of the present study and might support the validity of the results.

This study has several limitations. First, because the sample size of this study was relatively small due to this being a single-center study, the subjects of this study were not a representative population of schizophrenia patients in Japan. Second, treatment in this study was not controlled; there may be some treatment biases in this study. Third, because this study focused on doses of antipsychotics and BZPs, any other drugs, including anticholinergic drugs, which were reported to affect cognitive function in patients with schizophrenia were not evaluated. ${ }^{21}$ Fourth, several clinical characteristics of the subjects, some of which were also reported to be associated with relapse, were also not evaluated. ${ }^{2}$ Fifth, because subjects who were discharged between 2009 and 2012 were studied, the result of this study might not reflect the current clinical care situation. Sixth, because this study was not conducted by an intention-to-treat analysis and the entire discharged schizophrenia cohort was not included, the effect of BZP usage on rehospitalization might be biased by these methodological issues. Seventh, because this study was conducted using a retrospective design, a prospective and longitudinal study will be needed to confirm the relationship between continuing usage of benzodiazepine and rehospitalization.

\section{Conclusion}

In conclusion, high-dose use of BZPs, as well as younger onset age and lower educational history, in patients with schizophrenia might be associated with a shorter time to rehospitalization. In the future, a controlled study with a larger population will be required to confirm our findings. Clinicians should take care while prescribing higher doses of BZPs in order to avoid adverse events in the treatment of schizophrenia. 


\section{Acknowledgment}

We are indebted to the medical editors of the Department of International Medical Communications at Tokyo Medical University for editing and reviewing the initial English manuscript.

\section{Disclosure}

Yoshikazu Takaesu has received lecture fees from Otsuka Pharmaceutical, Meiji Seika Pharma, Eli Lilly, Eisai, Mitsubishi Tanabe Pharma, and Yoshitomi Pharmaceutical and has received research funding from Otsuka Pharmaceutical, Meiji Seika Pharma, and Eisai. Yuichi Inoue has received clinically pertinent fees, lecture fees, and research funding from Nippon Boehringer Ingelheim, Takeda Pharmaceutical, Astellas Pharma, Philips Respironics, Alfresa Pharma, MSD, Pacific Medico, Otsuka Pharmaceutical, Eisai, Yoshitomiyakuhin, and Hisamitsu Pharmaceutical. Takeshi Inoue has received honoraria from GlaxoSmithKline, Pfizer, Eli Lilly, Mitsubishi Tanabe Pharma, Mochida Pharmaceutical, Otsuka Pharmaceutical, Meiji Seika Pharma, Asahi Kasei Pharma, Shionogi, Dainippon Sumitomo Pharma, Takeda Pharmaceutical, MSD, Eisai, AbbVie GK, and Yoshitomiyakuhin; he has received research/grant support from Otsuka Pharmaceutical, Eli Lilly, Eisai, Mitsubishi Tanabe Pharma, Yoshitomiyakuhin, AbbVie GK, Pfizer, Astellas, MSD, and Meiji Seika Pharma; he is a member of the advisory boards of GlaxoSmithKline, Pfizer, Eli Lilly, Mochida Pharmaceutical, and Mitsubishi Tanabe Pharma. The authors report no other conflicts of interest in this work.

\section{References}

1. Emsley R, Chiliza B, Asmal L, Harvey BH. The nature of relapse in schizophrenia. BMC Psychiatry. 2013;13:50.

2. Leucht S, Tardy M, Komossa K, Heres S, Kissling W, Davis JM. Maintenance treatment with antipsychotic drugs for schizophrenia. Cochrane Database Syst Rev. 2012;5:CD008016.

3. Lacro JP, Dunn LB, Dolder CR, Leckband SG, Jeste DV. Prevalence of and risk factors for medication nonadherence in patients with schizophrenia: a comprehensive review of recent literature. J Clin Psychiatry. 2002; 63(10):892-909.

4. Schennach R, Obermeier M, Meyer S, et al. Predictors of relapse in the year after hospital discharge among patients with schizophrenia. Psychiatr Serv. 2012;63(1):87-90.
5. Boyer L, Millier A, Perthame E, Aballea S, Auquier P, Toumi M. Quality of life is predictive of relapse in schizophrenia. BMC Psychiatry. 2013; 13:15.

6. Lysaker PH, Bell MD, Bioty S, Zito WS. Performance on the Wisconsin Card Sorting Test as a predictor of rehospitalization in schizophrenia. J Nerv Ment Dis. 1996;184(5):319-321.

7. Haro JM, Novick D, Suarez D, Alonso J, Lépine JP, Ratcliffe M; SOHO Study Group. Remission and relapse in the outpatient care of schizophrenia: three-year results from the Schizophrenia Outpatient Health Outcomes study. J Clin Psychopharmacol. 2006;26(6):571-578.

8. Fennig S, Rabinowitz J, Fennig S. Involuntary first admission of patients with schizophrenia as a predictor of future admissions. Psychiatr Serv. 1999;50(8):1049-1052.

9. Eaton WW, Mortensen PB, Herrman H. Long-term course of hospitalization for schizophrenia: part I. Risk for rehospitalization. Schizophr Bull. 1992;18(2):217-228.

10. van der Hooft CS, Schoofs MW, Ziere G, et al. Inappropriate benzodiazepine use in older adults and the risk of fracture. Br J Clin Pharmacol. 2008;66(2):276-282.

11. Takaesu Y, Ishikawa J, Komada Y, et al. Prevalence of and factors associated with sleep-related eating disorder in psychiatric outpatients taking hypnotics. J Clin Psychiatry. 2016;77(7):e892-e898.

12. Murakoshi A, Takaesu Y, Komada Y, Ishikawa J, Inoue Y. Prevalence and associated factors of hypnotics dependence among Japanese outpatients with psychiatric disorders. Psychiatry Res. 2015;230(3): 958-963.

13. de las Cuevas C, Sanz E, de la Fuente J. Benzodiazepines: more "behavioural" addiction than dependence. Psychopharmacology (Berl). 2003;167(3):297-303.

14. Dold M, Li C, Gillies D, Leucht S. Benzodiazepine augmentation of antipsychotic drugs in schizophrenia: a meta-analysis and Cochrane review of randomized controlled trials. Eur Neuropsychopharmacol. 2013;23(9):1023-1033.

15. Kitajima R, Miyamoto S, Tenjin T, et al. Effects of tapering of long-term benzodiazepines on cognitive function in patients with schizophrenia receiving a second-generation antipsychotic. Prog Neuropsychopharmacol Biol Psychiatry. 2012;36(2):300-306.

16. Pfiffner C, Steinert T, Kilian R, et al. Rehospitalization risk of former voluntary and involuntary patients with schizophrenia. Soc Psychiatry Psychiatr Epidemiol. 2014;49:1719-1727.

17. Baandrup L, Gasse C, Jensen VD, et al. Antipsychotic polypharmacy and risk of death from natural causes in patients with schizophrenia: a population-based nested case-control study. J Clin Psychiatry. 2010; 71(2):103-108.

18. Marder SR, Soraburu S, Dunayevich E, et al. Case reports of postmarketing adverse event experiences with olanzapine intramuscular treatment in patients with agitation. J Clin Psychiatry. 2010;71(4):433-441.

19. Wulff K, Dijk DJ, Middleton B, Foster RG, Joyce EM. Sleep and circadian rhythm disruption in schizophrenia. Br J Psychiatry. 2012;200(4): 308-316.

20. Suzuki Y, Yasumura S, Fukao A, Otani K. Associated factors of rehospitalization among schizophrenic patients. Psychiatry Clin Neurosci. 2003; 57(6):555-561.

21. Ogino S, Miyamoto S, Tenjin T, et al. Effects of discontinuation of longterm biperiden use on cognitive function and quality of life in schizophrenia. Prog Neuropsychopharmacol Biol Psychiatry. 2011;35(1):78-83.

\section{Publish your work in this journal}

Neuropsychiatric Disease and Treatment is an international, peerreviewed journal of clinical therapeutics and pharmacology focusing on concise rapid reporting of clinical or pre-clinical studies on a range of neuropsychiatric and neurological disorders. This journal is indexed on PubMed Central, the 'PsycINFO' database and CAS,

\section{Dovepress}

and is the official journal of The International Neuropsychiatric Association (INA). The manuscript management system is completely online and includes a very quick and fair peer-review system, which is all easy to use. Visit http://www.dovepress.com/testimonials.php to read real quotes from published authors. 